\title{
Safe and Effective Use of Chronic Transdermal Estradiol for Life-Threatening Uremic Bleeding in a Patient with Coronary Artery Disease
}

\author{
Atul Balia John Kevin Hix ${ }^{a}$ b Peter Kouides $^{c}$ \\ a Department of Internal Medicine, Rochester General Hospital, b Nephrology Division, \\ Department of Medicine, University of Rochester School of Medicine and Dentistry, and \\ 'Division of Hematology/Oncology, Department of Medicine, University of Rochester \\ Medical Center, Rochester, N.Y., USA
}

\section{Key Words}

Uremic platelets $\cdot$ Transdermal estradiol $\cdot$ Chronic kidney disease $\cdot$ Bleeding

\begin{abstract}
Uremic platelet dysfunction rarely causes significant bleeding in adequately dialyzed patients. When encountered, the management is complicated by a lack of well-supported treatment modalities. Estrogen use in uremic platelet dysfunction has been described, but enthusiasm for the treatment has been dampened by the risk of thrombotic events in vasculopathic dialysis patients. We present a patient on long-term peritoneal dialysis with coronary disease who developed recurrent life-threatening bleeding episodes secondary to uremia, where treatment with transdermal estrogen was used safely and effectively for a 24-month period.
\end{abstract}

(C) 2014 S. Karger AG, Basel

\section{Introduction}

Bleeding from uremic platelet dysfunction causes significant morbidity in patients with chronic kidney disease, and treatment is complicated by our limited understanding of its exact pathophysiology and comorbid conditions [1-3]. Typical presentations include mucocutaneous or postprocedural bleeding $[1,2]$. While dialysis and improved anemia management with erythropoiesis-stimulating agents have greatly reduced the incidence of this condition, it has not yet been eliminated [1-3]. 
Table 1. Laboratory data on the days of admission along with preceding Kt/V

\begin{tabular}{|c|c|c|c|c|c|}
\hline Date & Hematocrit, \% & Platelets, $\times 10^{3} / \mu \mathrm{l}$ & INR & APTT, s & $\mathrm{Kt} / \mathrm{V}$ \\
\hline $2 / 15 / 2010$ & & & & & 2.23 \\
\hline $3 / 18 / 2010$ & 21 & 195 & 1.0 & 24.5 & \\
\hline $4 / 9 / 2010$ & 22 & 355 & 1.2 & & \\
\hline $4 / 23 / 2010$ & 27 & 358 & & & \\
\hline $6 / 4 / 2010$ & 34 & 241 & 1.0 & 23.0 & \\
\hline $7 / 8 / 2010$ & & & & & 1.5 \\
\hline $7 / 26 / 2010$ & 33 & 291 & 1.0 & 26.7 & \\
\hline $8 / 4 / 2010$ & 25 & 260 & 1.0 & 27.5 & \\
\hline $8 / 11 / 2010$ & 27 & 327 & 1.0 & 27.8 & \\
\hline $9 / 1 / 2010$ & 32 & 339 & 1.0 & 26.9 & \\
\hline $9 / 24 / 2010$ & & & & & 2.3 \\
\hline
\end{tabular}

APTT $=$ Activated partial thromboplastin time.

We report a patient with end-stage renal disease who suffered multiple spontaneous lifethreatening bleeding episodes due to uremic platelet dysfunction responsive only to transdermal estrogen.

\section{Case Report}

A 79-year-old male with end-stage renal disease secondary to hypertension presented to the emergency room with lightheadedness. He had been on regular peritoneal dialysis for the preceding 18 months. Five days prior to presentation he had had a tooth extraction complicated by profuse daily bleeding at the wound site. His history was notable for coronary artery disease with coronary artery bypass graft surgery in 1996 and atrial fibrillation. He reported no prior personal or family history of bleeding. His medications included aspirin (81 $\mathrm{mg}$ ) and warfarin. Laboratory data revealed a subtherapeutic INR (1.0) and severe anemia (hemoglobin $7.1 \mathrm{~g} / \mathrm{dl}$ ). He received 3 units of packed red blood cells (PRBC) and was discharged having been taken off warfarin.

Three weeks later, the patient presented with acute-onset right hip pain from a spontaneous right iliacus hematoma $(7.9 \times 6.3 \mathrm{~cm})$ and severe anemia requiring multiple PRBC transfusions (table 1). Aspirin was discontinued but was restarted shortly after discharge. Six weeks after resumption, he developed a spontaneous left psoas hematoma $(7.2 \times 14.6 \mathrm{~cm})$ and aspirin was permanently discontinued. Over the next 6 months, he required 8 hospital admissions and 25 units of PRBC due to repeated spontaneous gluteal hematomas while being off all anticoagulant and antiplatelet therapies. Dialysis was usually adequate based on urea kinetics calculated during the periods between admissions (table 1). During admissions, peritoneal dialysis was extended up to $14 \mathrm{~h}$ daily to facilitate toxin removal. His hematocrit level was maintained close to $30 \%$ by means of PRBC transfusions to ensure cell availability for 'scaffolding' of the vascular endothelium by platelets for optimal functionality. Darbepoetin use was continued in both the inpatient and outpatient setting. Desmopressin was employed once but was subsequently abandoned due to its lack of efficacy and the possibility of tachyphylaxis. None of these measures prevented the formation of new hematomas.

Hematologic workup during this period showed that PT/INR, activated partial thromboplastin time, platelet count, factors VIII, IX, XI and XIII, fibrinogen, euglobulin clot lysis time 
Bali et al.: Safe and Effective Use of Chronic Transdermal Estradiol for Life-Threatening Uremic Bleeding in a Patient with Coronary Artery Disease

and vitamin $\mathrm{C}$ levels were within normal limits. High levels of von Willebrand factor antigen (447\%, normal range $60-150 \%)$ and ristocetin cofactor (542\%, normal range $50-175 \%$ ) were noted. Platelet function testing on whole blood revealed low release to thrombin and low-dose collagen, borderline low release to high-dose collagen and adenosine diphosphate, and normal release to arachidonic acid. Normal aggregation was observed to all agonists. Based on these findings, platelet dysfunction was diagnosed, which, with no other identifiable cause present, was deemed secondary to uremia.

The patient experienced such deterioration in quality of life that he even considered stopping dialysis and electing only palliative treatment. After multidisciplinary discussions and with informed consent, the patient was offered transdermal estradiol patches starting at $50 \mu \mathrm{g} /$ day once every 3.5 days [4]. The transdermal route was offered based on data suggesting an association with lower thrombotic risk [5, 6]. Treatment was started in September 2010. After a 16-month period of complete clinical stability, the dose was reduced to $25 \mu \mathrm{g} /$ day every 3.5 days. Over a total treatment time of 25 months using estradiol patches, the patient suffered no further bleeding or new cardiac events. Repeat CT scans of the abdomen in February 2012 and July 2012 confirmed the resolution of the previous retroperitoneal hematomas without new findings. Recently, the patient has elected to come off treatment on the recommendation of his cardiology team.

\section{Discussion}

The mainstay of preventing uremic platelet dysfunction is regular adequate dialysis to reduce the toxic milieu that impairs platelet function and to improve their functionality. Evidence suggests that peritoneal dialysis may have a greater influence on platelet aggregation than hemodialysis [3].

For patients with persisting platelet dysfunction despite dialysis, conjugated estrogens have been utilized $[4,7]$. The knowledge of their efficacy and mechanism of action is based on several small studies conducted in the 1980s. The proposed mechanism is attributed to the effect of conjugated estrogens on reducing L-arginine levels, thereby reducing the production of nitric oxide, which is an inhibitor of platelet aggregation $[1,3]$. Consequently, estrogens promote platelet reactivity by increasing the serum levels of $\beta$-thromboglobulin and thromboxane $[2,3,7]$. Evidence suggests that their effects on bleeding time and clinical bleeding are dose dependent [8].

The risk of thrombotic events associated with the use of estrogen in elderly dialysis patients with cardiovascular disease is a major concern, and the long-term effects of transdermal estrogens have not yet been studied in this population. The typical dose of estrogen used for uremic bleeding is comparable to the dosages used for the purpose of hormone replacement in menopausal women. A systematic review by Hemelaar et al. [5] supported the safety of nonoral routes of estrogen administration for postmenopausal hormone replacement therapy compared to oral estrogens, with respect to the risk of developing atherosclerotic and venous thromboembolic disease. A meta-analysis conducted by Olié et al. [6] further supported these findings. Potentially, this route avoids the first-pass metabolism that oral estrogens undergo, which may subsequently increase the hepatic synthesis of inflammatory markers and alter the production of proteins participating in hemostasis [4, 6]. In addition, an increased incidence of acquired activated protein $\mathrm{C}$ resistance has been described in oral but not in transdermal estrogen users [6]. In light of this information, the European Menopause and Andropause Society favors the transdermal route in patients who require estrogen therapy because they are suffering from coronary disease or are at high risk for venous thromboembolic disease $[9,10]$. 
Bali et al:: Safe and Effective Use of Chronic Transdermal Estradiol for Life-Threatening Uremic Bleeding in a Patient with Coronary Artery Disease

It is premature to extrapolate these results to patients with uremic platelet dysfunction. However, the long-term success and safety of using transdermal estrogen outlined in this report are encouraging and warrant consideration of this option in treating uremic platelet dysfunction.

\section{Disclosure Statement}

The authors received no financial support for this work and declare no competing interests.

\section{References}

1 Noris M, Remuzzi G: Uremic bleeding: closing the circle after 30 years of controversies? Blood 1999;94:2569_ 2574.

2 Pavord S, Myers B: Bleeding and thrombotic complications of kidney disease. Blood Rev 2011;25:271-278.

-3 Hedges SJ, Dehoney SB, Hooper JS, et al: Evidence-based treatment recommendations for uremic bleeding. Nat Clin Pract Nephrol 2007;3:138-152.

4 Sloand JA, Schiff MJ: Beneficial effect of low-dose transdermal estrogen on bleeding time and clinical bleeding in uremia. Am J Kidney Dis 1995;26:22.

5 Hemelaar M, van der Mooren M, Rad M, et al: Effects of non-oral postmenopausal hormone therapy on markers of cardiovascular risk: a systematic review. Fertil Steril 2008;90:642-672.

6 Olié V, Canonico M, Scarabin PY: Risk of venous thrombosis with oral versus transdermal estrogen therapy among postmenopausal women. Curr Opin Hematol 2010;17:457-463.

7 Livio M, Mannuccio PM, Viganò G, et al: Conjugated estrogens for the management of bleeding associated with renal failure. N Engl J Med 1986;315:731.

-8 Viganò G, Gaspari F, Locatelli M, et al: Dose-effect and pharmacokinetics of estrogens given to correct bleeding time in uremia. Kidney Int 1988;34:853.

$\rightarrow 9$ Tremollieres F, Brincat M, Erel CT, et al: EMAS position statement: managing menopausal women with a personal or family history of VTE. Maturitas 2011;69:195-198.

10 Schenck-Gustafsson K, Brincat M, Erel CT, et al: EMAS position statement: managing the menopause in the context of coronary heart disease. Maturitas 2011;68:94-97. 